\section{ORIGINAL} RESEARCH

\author{
B.M. Ellingson \\ J.L. Ulmer \\ S.N. Kurpad \\ B.D. Schmit
}

\title{
Diffusion Tensor MR Imaging in Chronic Spinal Cord Injury
}

BACKGROUND AND PURPOSE: Diffusion tensor MR imaging is emerging as an important tool for displaying anatomic changes in the brain after injury or disease but has been less widely applied to disorders of the spinal cord. The aim of this study was to characterize the diffusion properties of the entire human spinal cord in vivo during the chronic stages of spinal cord injury (SCI). These data provide insight into the structural changes that occur as a result of long-term recovery from spinal trauma.

\begin{abstract}
MATERIALS AND METHODS: Thirteen neurologically intact subjects and 10 subjects with chronic $\mathrm{SCl}$ ( $>4$ years postinjury) were enrolled in this study. A single-shot twice-refocused spin-echo diffusionweighted echo-planar imaging pulse sequence was used to obtain axial images throughout the entire spinal cord (C1-L1) in $<60$ minutes.
\end{abstract}

RESULTS: Despite heterogeneity in SCl lesion severity and location, diffusion characteristics of the chronic lesion were significantly elevated compared with those of uninjured controls. Fractional anisotropy was significantly lower at the chronic lesion and appeared dependent on the completeness of the injury. Conversely, mean diffusivity measurements in the upper cervical spinal cord in subjects with $\mathrm{SCl}$ were significantly lower than those in controls. These trends suggest that the entire neuraxis may be affected by long-term recovery from spinal trauma.

CONCLUSION: These results suggest that diffusion tensor imaging may be useful in the assessment of $\mathrm{SCl}$ recovery.

D iffusion tensor imaging (DTI) has been successfully used to characterize structural changes in neural tissue after spinal artery stroke, ${ }^{1}$ multiple sclerosis, ${ }^{2,3}$ cervical spondylotic myelopathy, ${ }^{4}$ spinal cord compression, ${ }^{5}$ and acute spinal cord injury $(\mathrm{SCI})^{6,7}$; however, DTI has not been used to explore the long-term changes in spinal cord structure known to accompany chronic SCI..$^{8-11}$ The purpose of this study was to characterize the diffusion values of the entire spinal cord in humans with chronic SCI $(>4$ years postinjury) by using a clinically available pulse sequence and comparing these data with normative DTI characteristics reported previously. ${ }^{12}$ On the basis of previous work, ${ }^{13}$ we hypothesized that diffusion characteristics would be significantly altered throughout the entire length of the spinal cord.

DTI research in SCI largely involves the use of experimental animal models to examine changes in diffusivity that accompany the early stages of injury. These studies have suggested overall diffusivity increases and diffusion anisotropy decreases near the injury site ${ }^{14-18}$ due to axonal damage and/or vasogenic edema. ${ }^{15}$ Although a few studies have been conducted with human spinal cord pathologies, ${ }^{4-7,19}$ they have also demonstrated an increase in diffusivity and a decrease in diffusion anisotropy.

DTI may be useful for identifying the characteristics of chronic SCI, because structural changes in the spinal cord during the chronic stages may differ from the normal spinal cord and the spinal cord in acute stages of injury. For example,

Received June 9, 2008; accepted June 27.

From the Department of Biomedical Engineering (B.M.E., B.D.S.), Marquette University, Milwaukee, Wis; and Departments of Radiology (J.L.U.) and Neurosurgery (S.N.K.), Medical College of Wisconsin, Milwaukee, Wis.

Please address correspondence to Brian D. Schmit, PhD, Marquette University, Department of Biomedical Engineering, PO Box 1881, Milwaukee, WI 53201-1881; e-mail: brian.schmit@marquette.edu

DOI 10.3174/ajnr.A1272 extensive longitudinal spreading of lesions in the late stages of injury creates widespread changes in the spinal cord morphology, including cyst formation and necrosis. ${ }^{20}$ Changes in diffusivity associated with these structural alterations may make it possible to identify the rostral and caudal extent of a spinal lesion by using DTI. Many therapeutic interventions for rehabilitation after SCI, including functional electric stimulation ${ }^{21}$ and gait training, ${ }^{22}$ rely on intact spinal motoneurons below the level of the lesion. Incomplete injury in segments below the injury may be particularly difficult to identify by using physical or electrophysiologic measurements because sensory and motor function is often reduced or absent below the level of injury. Thus, DTI provides an opportunity to assess the integrity of the spinal cord.

DTI might also be sensitive to changes in the structure of the spinal cord tissue in regions distant from the spinal lesion in chronic injury. Although the chronic stages of SCI are typically considered stable, ${ }^{23,24}$ progressive demyelination in chronic SCI has been documented, ${ }^{8-10}$ and remyelination, when it occurs, can result in significantly decreased myelin sheath thickness ${ }^{8,25-27}$ and preferential loss of largediameter axons. ${ }^{26}$ Also, considerable atrophy of the spinal cord occurs in the late stages of SCI, causing the remaining axons to be compressed and tightly packed. ${ }^{11}$ These changes could increase the attenuation of diffusion barriers, which would be consistent with the decrease in mean diffusivity recently reported in the upper cervical spinal cord rostral to the injury in a small number of subjects with chronic SCI. ${ }^{13}$

Thus, the primary aim of this study was to characterize the diffusion properties across the entire spinal cord (C1-L1 vertebral levels) in humans with chronic SCI by using a clinically available DTI pulse sequence. We then compared these data with diffusion characteristics from a previously published young neurologically intact sample. ${ }^{12}$ 


\begin{tabular}{lcc}
\hline \multicolumn{2}{c}{ Table 1: Subjects with chronic $\mathbf{S C I}$} \\
\hline No. & ASIA Injury & Completeness \\
\hline S1 & C7-T1 & Complete \\
S2 & T2 & Incomplete \\
S3 & C5-C7 & Incomplete \\
S4 & C5 & Complete \\
S5 & T12 & Incomplete \\
S6 & C5-C6 & Incomplete \\
S7 & T4-T11 & Complete \\
S8 & C5-C7 & Complete \\
S9 & T6 & Incomplete \\
S10 & C5-C6 & Incomplete \\
\hline
\end{tabular}

Note:-SCl indicates spinal cord injury; ASIA, American Spinal Injury Association.

\section{Materials and Methods}

\section{Subjects}

Thirteen neurologically intact subjects (age range, 19-40 years; median age, 25 years) and 10 subjects with chronic SCI (age range, 25-67 years; median age, 37 years) having an injury for $>4$ years participated in this study. Control subjects had no history of neurologic illness, and all subjects except 1 were free of medical implants. One subject with SCI, S9, had vertebral fixation, which substantially lowered image quality; thus, only measurements above the lesion site were used. The neurologic level of injury was determined by the American Spinal Injury Association (ASIA) scale (available at: http://www.asiaspinalinjury. org/publications/2006_Classif_worksheet.pdf), which was used as the basis for defining the lesion site and completeness of injury (complete versus incomplete SCI). Table 1 shows the characteristics of the subjects with chronic SCI. The neurologically intact population used for comparison was from a previous publication. ${ }^{12}$ Although subjects with SCI were not age-matched and our control population was relatively young, the magnitude of diffusion and anisotropy differences due to age variation was expected to be minimal ${ }^{4}$ compared with changes as a result of SCI. The possible effects of subject age are addressed in more detail within the "Discussion" section. All procedures complied with the principles of the Declaration of Helsinki and were approved by the Institutional Review Boards of Marquette University and the Medical College of Wisconsin.

\section{MR Imaging}

The imaging procedures were similar to those described previously for imaging the spinal cord in uninjured controls. ${ }^{12}$ Briefly, DTI was performed axially throughout the entire spinal cord (C1-L1) by using a clinically available single-shot twice-refocused spin-echo echo-planar (EPI) pulse sequence. ${ }^{28}$ This particular pulse sequence has been shown to improve image quality significantly and reduce susceptibility distortions. ${ }^{28}$ A CTL Spine Coil (GE Healthcare, Milwaukee, Wis) with an anterior neck coil attachment and a $1.5 \mathrm{~T}$ clinical MR imaging scanner (Signa Excite; GE Healthcare) were used for all image acquisitions. Spinal levels were identified by using sagittal fast low-angle shot (FLASH) locator MR images based on known anatomic landmarks. Images were acquired with TE/TR $=96.3 / 6000 \mathrm{~ms}$, matrix size $=128 \times 128, \mathrm{NEX}=1, \mathrm{FOV}=200 \mathrm{~mm}$, and a section thickness of $5 \mathrm{~mm}$ with no intersection gap. After reconstruction, images were zero-padded and interpolated to $256 \times 256$. Diffusion-weighted images (DWIs) were acquired with $b=1500 \mathrm{~s} / \mathrm{mm}^{2}$ in 25 equidistant directions, and a single T2-weighted (T2WI) $\left(b=0 \mathrm{~s} / \mathrm{mm}^{2}\right)$ image was acquired for each section. The number of diffusion-sensitizing directions was chosen on the basis of previous studies illustrating no significant increase in image quality for $>25$ directions. ${ }^{29,30}$

Analysis of Functional Neuroimages software package (AFNI; available at http://afni.nimh.nih.gov/) was used for Fourier transform-based affine registration of the 25 DWIs to the T2WI reference image to correct for bulk motion, eddy-currents, and susceptibilityrelated image distortions. AFNI was also used to calculate the $3 \times 3$ diffusion tensor from the 25 DWIs by using a constrained nonlinear least squares approximation. ${ }^{31}$ The eigenvalues $\left(\lambda_{1}, \lambda_{2}, \lambda_{3}\right)$, transverse apparent diffusion coefficient $\left(\lambda_{\mathrm{t}}\right.$, defined as the average of the 2 smaller eigenvalues, $\lambda_{2}$ and $\lambda_{3}$ ), longitudinal apparent diffusion coefficient $\left(\lambda_{1}\right.$, defined as the largest eigenvalue, or $\left.\lambda_{1}\right)$, eigenvectors $\left(\nu_{1}\right.$, $\nu_{2}, \nu_{3}$ ), mean diffusivity (MD, defined as average of $\lambda_{1}, \lambda_{2}$, and $\lambda_{3}$ ), and fractional anisotropy (FA, see ${ }^{32}$ for equation) were calculated for each voxel by using custom Matlab (MathWorks, Natick, Mass) algorithms. A total of 108-126 sections were obtained throughout the entire spinal cord, resulting in a total scanning time of $48 \pm 12 \mathrm{~min}$ utes, depending on the height of the subject. Individual subject scanning times were determined from the start of the first FLASH locator image to the last DWI image.

\section{Data Description for Regions of Interest}

Manual segmentation of spinal cord regions of interest was performed for the whole cord as described in a previous publication. ${ }^{12}$ First, the spinal cord was manually segmented from the CSF by using the T2WI images for a whole cord analysis (ie, no gray and white matter segmentation). Regions of interest for the whole cord analysis were placed within the spinal cord such that at least 2 voxels around the edge of the cord were excluded, to ensure no partial volume contamination from the surrounding CSF. Manual segmentation of the spinal cord was verified by comparison with an accepted automated segmentation algorithm. ${ }^{13,33}$

For data analysis with respect to vertebral level, extracted diffusion distributions from all sections within a single vertebral level (approximately 3 sections per vertebral level) were pooled into a single representative distribution per segment. For data analysis with respect to distance from chronic lesions, extracted distributions from each section were used to represent the diffusion characteristics at the particular section location. For example, the diffusion characteristics 15 $\mathrm{mm}$ from a chronic lesion located at C5 corresponded to 3 sections from the image located at the center of the $\mathrm{C} 5$ vertebral body (because section thickness was $5 \mathrm{~mm}$ ).

\section{Hypothesis Testing and Statistics}

Whole Cord Diffusion Characteristics. FA and diffusion characteristics were analyzed for all subjects and all vertebral levels to determine if global changes in diffusivity occur along the neuraxis during the chronic stages of SCI. Tests for normality (Kolmogorov-Smirnov test) were performed before analysis to ensure that parametric statistics were applicable. If data were normally distributed $(P>.05)$, a 2 -way analysis of variance (ANOVA) with pooled variance (fixed factors: injury group [injured/uninjured], vertebral level; random factor: subject) was performed to compare injured subjects with uninjured subjects. In addition, a univariate general linear model (GLM; fixed factors: vertebral level, completeness of injury [complete/incomplete], injury level [thoracic/cervical], vertebral level $\times$ injury level, vertebral level $\times$ completeness of injury; random factor: subject) was performed to examine differences between injured subject groups. The Tukey test for multiple comparisons was performed for post hoc comparisons. All tests had a significance level of $\alpha=.05$.

Chronic Lesion Diffusion Characteristics. To characterize the diffusivity in regions near the chronic lesion across SCI subjects, we aligned diffusion data longitudinally, on the basis of the level of neu- 
rologic impairment. Diffusion measurements $\left(\lambda_{1}, \lambda_{\mathrm{t}}\right.$, and MD) of the chronic lesion were examined to determine the nature of water diffusion as a function of distance or section number from the injury. A length of $100 \mathrm{~mm}$, centered on the chronic lesion, was examined in this portion of the study. Diffusion measurements within the chronic lesion were compared with a similar volume of spinal cord (100-mm segment) in the uninjured cervical (approximately C1-C7) and thoracic regions of controls (approximately T3-T9), which were previously determined to be significantly different. ${ }^{12}$ Again, 2 separate 2-way ANOVAs with pooled variance (fixed factors: distance, injury group [injured/uninjured]; random factor: subject) were used to determine if significant differences in diffusion measurements existed between the lesion and uninjured cervical or thoracic spinal cord. In addition, a univariate GLM (fixed factors: distance, completeness of injury [complete/incomplete], level of injury [thoracic/cervical], distance $\times$ completeness, distance $\times$ level of injury; random factor: subject) was performed to test differences between injured subject groups and to determine the effects of lesion location and completeness of injury on diffusion measurements.

Trends in FA adjacent to and at gradually increasing vertebral levels from the lesion site were then examined. If data were normally distributed, a 2-way ANOVA with pooled variance (fixed factors: vertebral level, injury group [injured/uninjured]; random factor: subject) was used to compare FA in injured subjects with either the uninjured cervical (C1-C7) or thoracic (T2-T7) spinal cord. A univariate GLM (fixed factors: vertebral level, completeness of injury [complete/incomplete], injury level [thoracic/cervical], vertebral level $\times$ completeness, vertebral level $\times$ injury level; random factor: subject) was performed to examine differences between injured subject groups and to determine the effects of lesion location and completeness of injury on diffusion measurements. The Tukey test for multiple comparisons was performed for post hoc analyses. Significance level was set to $\alpha=.05$ for all tests.

Diffusion Characteristics of the Upper Cervical Spinal Cord. To examine whether diffusion properties differ in regions remote from the injury site, as previously suggested, ${ }^{13}$ we used a 2 -way ANOVA with pooled variance (fixed factors: vertebral level, injury group [injured/uninjured]; random factor: subject) and the Tukey test to compare the diffusion characteristics $\left(\lambda_{1}, \lambda_{t}\right.$, and MD) in the upper cervical spinal cord (C1-C3) between uninjured subjects and those with chronic SCI. In addition, a univariate GLM (fixed factors: completeness of injury [complete/incomplete], level of injury [thoracic/cervical], vertebral level [C1-C3]; random factor: subject) was performed to examine differences between injury groups and the effects of lesion level and completeness of injury on cervical measurements of diffusivity.

\section{Results}

The T2WI signal-intensity-to-noise ratio (SNR) was found to be approximately $5.7 \pm 0.7$ but varied across the length of the spinal cord. ${ }^{12}$ Specifically, upper cervical levels had significantly higher SNR compared with midthoracic regions (C1$\mathrm{C} 4, \mathrm{SNR}=6.5$; T2-T5, SNR $=4.5$; Tukey, $P<.05)$. No significant differences in SNR were observed between individual subjects (ANOVA, $P=.585$ ) or subject groups (ANOVA, $P=$ .312). The SNR was calculated as the mean T2WI signal intensity within the spinal cord divided by the mean T2WI signal intensity of background noise in the image. ${ }^{34}$

FA images in uninjured controls showed high anisotropy throughout the length of the spinal cord compared with that in subjects with SCI, especially in regions associated with spinal white matter. An example of the FA images in the upper cervical segments in a single control subject is illustrated in Fig $1 A$. In subjects with incomplete chronic SCI (Fig $1 B$ ), a lower FA was apparent compared with intact subjects. Subjects with complete chronic SCI, illustrated in Fig $1 C$, had very low FA values in regions near the injury site.

\section{Whole Cord Diffusion Characteristics}

Fluctuations in diffusion measurements were observed across the entire length of the spinal cord in subjects with chronic SCI, suggesting that widespread changes in spinal tissue structure may occur during long-term recovery from traumatic injury. Specifically, spinal cord diffusion measurements in subjects with chronic SCI illustrated a significant increase in $\lambda_{1}, \lambda_{\mathrm{t}}$, and $\mathrm{MD}$ at the lesion (Fig $2 A-C$ ), compared with diffusion measurements of neurologically intact control subjects (ANOVA, $P<.05$ for all $\lambda_{1}, \lambda_{\mathrm{t}}$, and MD comparisons). No differences between injured subject groups, completeness of injury, or lesion level were observed with respect to $\lambda_{1}$ (vertebral level, $P=.410$; injury level, $P=.068$; completeness, $P=$ .377; vertebral level $\times$ injury level, $P=.092$; vertebral level $\times$ completeness, $P=.539)$. Subjects with complete SCI had significantly higher $\lambda_{\mathrm{t}}(\mathrm{GLM}, P=.011)$ and $\mathrm{MD}(\mathrm{GLM}, P=.037)$ throughout the spinal cord compared with subjects with incomplete SCI. Subjects with thoracic lesions also demonstrated significantly higher $\lambda_{\mathrm{t}}(\mathrm{GLM}, P=.012)$ and $\mathrm{MD}$ (GLM, $P=.019$ ) throughout the spinal cord compared with lower cervical lesions. Interactions between lesion level and vertebral level along with the interactions between completeness of injury and vertebral level were not significant (GLM, $P>.05)$.

When examining group differences in FA between uninjured subjects and those with chronic SCI, results indicated a significantly lower FA in subjects with chronic SCI compared with controls (ANOVA, $P<.001$ ). The combined group analysis also indicated significant differences due to vertebral level (ANOVA, $P<.001$ ) and significant interaction effects (ANOVA, $P=.024$ ). The interaction effects (vertebral level and injury/control group) reflected low FA values for the SCI group at specific vertebral levels, which corresponded precisely to the level of ASIA neurologic impairment. In addition, subjects with complete SCI had significantly lower FA (GLM, $P<.001$ ) throughout the spinal cord compared with subjects with incomplete SCI. Subjects with thoracic injury had significantly lower FA (GLM, $P=.011$ ) compared with subjects who had lower cervical spinal cord lesions.

The potential utility of FA for identifying injury location is demonstrated for 1 subject with SCI in Fig 2D. Subject S6 demonstrated a relatively sharp drop in FA around the clinically diagnosed level of injury (C5-C6 incomplete), along with a rise in FA to that of control levels caudal to the injury. A similar pattern was observed in all injured subjects across the length of the spinal cord, where the sharp drop in FA corresponded precisely to the clinically diagnosed level of injury.

\section{Chronic Lesion Diffusion Characteristics}

Diffusion measurements of the subjects with chronic SCI lesions showed a significant increase in $\lambda_{1}(\sim 50 \%$ increase $), \lambda_{t}$ $(\sim 160 \%$ increase $)$, and MD $(\sim 100 \%$ increase $)$ at the lesion 


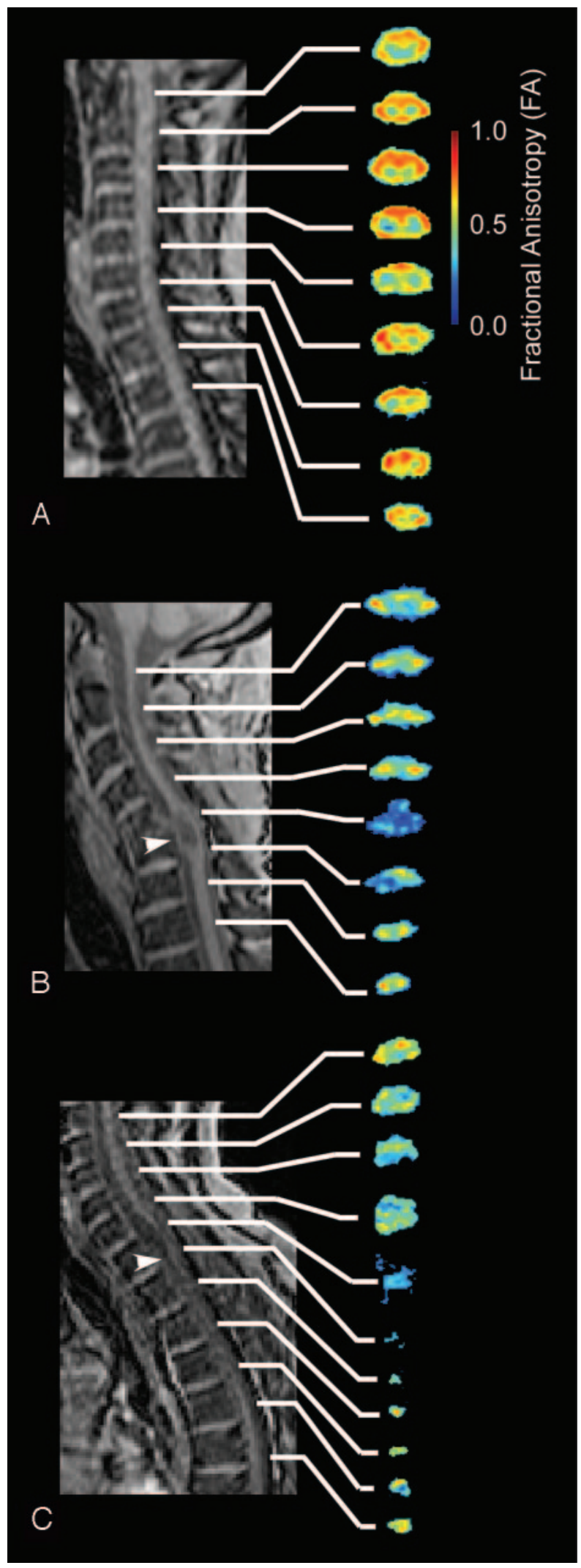

Fig 1. FLASH localizer and FA color images of select regions of the spinal cord in representative subjects. $A$, Control subject showing consistently high FA throughout the spinal cord. $B$, Representative subject with incomplete injury (S2) showing slightly lower FA compared with control subjects. Note that the level of the lesion had a particularly low FA value (arrowhead). C, Representative subject with complete injury (S4) showing significantly lower FA compared with that of the control subject in A. Note significant atrophy of the spinal cord caudal to the injury site.
(Fig 3A-C), compared with diffusion measurements in comparable cervical and thoracic volumes in neurologically intact controls (ANOVA, $P<.05$ for all $\lambda_{\mathrm{l}}, \lambda_{\mathrm{t}}$, and MD comparisons with both cervical and thoracic volumes). Subjects with complete and incomplete SCI did not have significantly different measures of $\lambda_{1}$ (GLM, completeness of injury, $P=.296$ ) or MD (GLM, completeness of injury, $P=.097$ ). In addition, level of injury was not a significant factor for $\lambda_{1}$ (GLM, injury level, $P=.483$ ) or MD (GLM, injury level, $P=.222$ ) near the lesion. Alternatively, $\lambda_{\mathrm{t}}$ illustrated a significant difference between subjects with complete and incomplete SCI (GLM, completeness of injury, $P=.009$ ) and level of injury (GLM, injury level, $P=.050$ ).

Around the site of traumatic lesions, FA was significantly lower compared with neurologically intact cervical (ANOVA, $P<.001$ ) and thoracic (ANOVA, $P<.001$ ) vertebral levels. Measurements of FA around the lesion site also appeared to be dependent on the level of injury (GLM, injury level, $P=.027$ ) as well as on the completeness of injury (GLM, completeness of injury, $P=.007$ ). Specifically, subjects having complete SCI demonstrated a lower FA at the lesion compared with subjects with incomplete SCI, and subjects with thoracic lesions had a lower FA compared with subjects having lower cervical lesions.

\section{Diffusion Characteristics of the Upper Cervical Spinal Cord}

The differences in diffusion measurements between subjects with SCI and controls were not constrained to regions of the spinal cord near the injury site. A comparison of the diffusion measurements in the upper cervical spinal cord (C1-C3) indicated significantly smaller magnitudes of diffusion in subjects with chronic SCI compared with those with an intact spinal cord (ANOVA, $P<.001$ for $\lambda_{1}, \lambda_{\mathrm{t}}$, and MD, Table 2 ). Completeness of injury and level of injury were not significant factors in determining $\lambda_{1}, \lambda_{\mathrm{t}}$, or MD in the cervical spinal cord (GLM, $P>.05$ for all $\lambda_{1}, \lambda_{\mathrm{t}}$, and MD). Subjects with complete SCI appeared to have significantly lower cervical cord FA compared with those with incomplete SCI (GLM, $P<.001)$; however, level of injury was not a significant factor (GLM, injury level, $P=0.171$ ). Note that this decrease in overall diffusivity in subjects with SCI is in marked contrast to the increase observed at and near the injury site. These results suggest that systemic changes in diffusivity throughout the entire spinal cord may occur as a result of long-term recovery from traumatic SCI.

\section{Discussion}

This study demonstrated changes in diffusion characteristics throughout the spinal cord in subjects with chronic SCI compared with uninjured controls. FA was particularly low at the injury site, providing a metric for estimation of the rostral and caudal extent of the damaged tissue. Furthermore, overall diffusion magnitude within the spinal cord was higher at the injury site but significantly reduced in the upper cervical segments, suggesting that regions remote from the injury site may be affected by the pathophysiologic processes associated with long-term recovery from spinal injury. Together, these observations demonstrate the utility of DTI for monitoring alterations in the spinal cord after injury. 

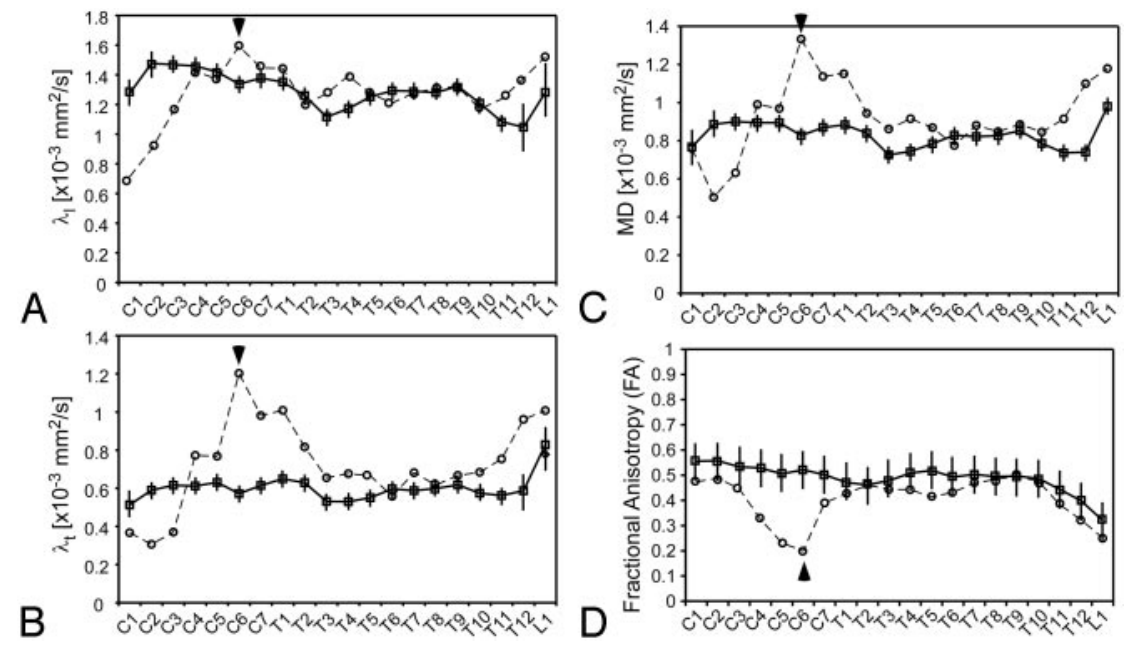

Fig 2. Whole cord diffusion characteristics for healthy subjects (mean for all healthy subjects \pm SEM across healthy subjects, illustrated with boxes) and a representative subject with chronic SCI (S6, incomplete [circles]). Longitudinal diffusivity $(\lambda l)(A)$, transverse diffusivity $(\lambda t)(B)$, mean diffusivity $(\mathrm{MD})(C)$, and FA $(D)$ are shown. Arrowheads represent the location of ASIA neurologic impairment for the subject with chronic SCI.

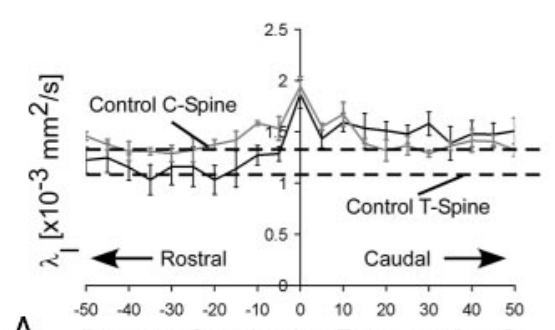

A

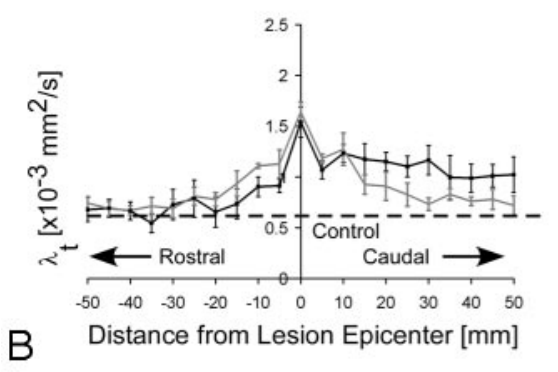

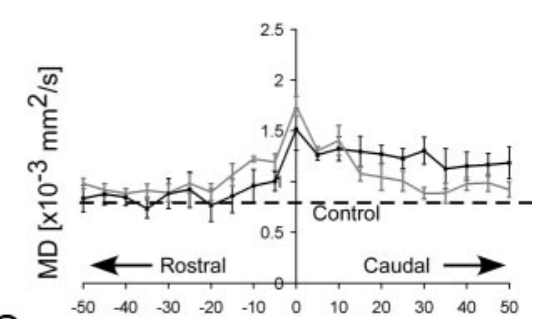

C

Distance from Lesion Epicenter [mm]

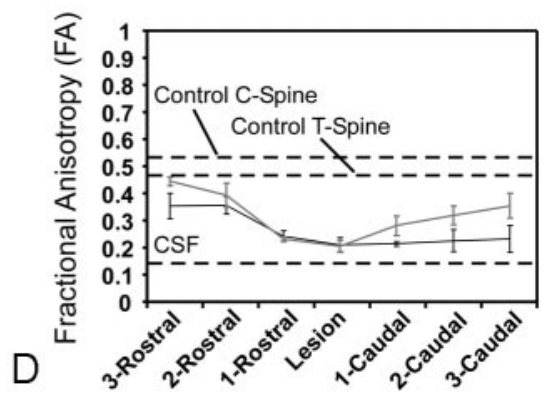

Fig 3. Mean diffusion measurements across lesions for all subjects with chronic $S C l$. $A-C$, Longitudinal diffusivity $(\lambda l)(A)$, transverse diffusivity $(\lambda t)(B)$, and mean diffusivity (MD) $(C)$ are shown as a function of distance (in millimeters) from the location of ASIA neurologic impairment. D, FA across the lesion is shown as a function of vertebral level. The black line represents clinically complete SCI, and gray lines represent clinically incomplete SCl. Dashed lines represent mean control data. Data shown represent mean values \pm SEM across SCI subjects (complete, $n=4$; incomplete, $n=5$ ).

The reduction in FA measurements in chronic SCI is consistent with decreases in FA reported at the injury site in animal models. In the current study, FA measurements throughout the entire spinal cord demonstrated a significant decrease in diffusion anisotropy in the chronically injured compared with the intact spinal cord. This observation is consistent with changes occurring during acute SCI in animal models ${ }^{14-17,35}$ and reports in humans with nontraumatic ${ }^{4,5,19}$ or traumatic spinal cord pathology. ${ }^{7}$ We observed that the chronic lesion, in particular, had dramatically lower FA compared with the neurologically intact spinal cord, and this decrease depended on the completeness of injury. Thus, measurements of spinal cord FA may be useful to indicate the site of the chronic lesion and degree of neural injury.

An important result of the current study was the observed decrease in $\lambda_{1}, \lambda_{\mathrm{t}}$, and MD in the upper cervical segments of subjects with chronic SCI compared with control subjects. Previous reports have consisted almost exclusively of increases in diffusivity after SCI. However, injuries in these studies have all been relatively acute, and measurements were isolated to the injury site (not in regions remote from the lesion). Conversely, a decrease in overall diffusion magnitude has been observed distant from the injury site in the sea lamprey during recovery from a spinal cord injury ${ }^{36}$ and in the upper cervical cord of 4 subjects with chronic SCI. ${ }^{13}$ In addition, a decrease in $\lambda_{1}$ distant from the primary lesion was also reported very recently in a rat model of focal myelitis, associated with axon degeneration. ${ }^{37}$ These studies are consistent with our results suggesting that the decrease in diffusion magnitude observed distal to the injury site is likely due to axonal restructuring and long-term widespread degeneration of the spinal cord.

The decreases in overall diffusion magnitude in the upper 


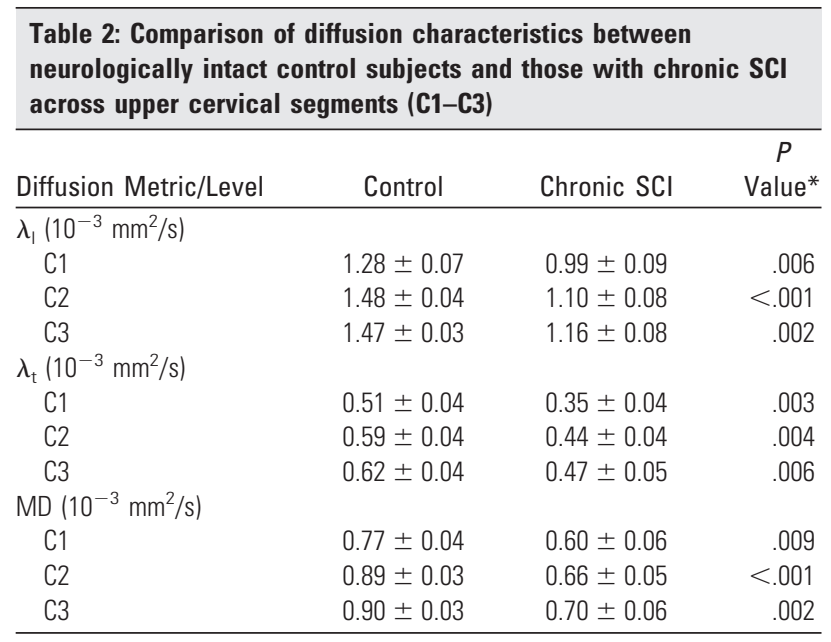

Note:-MD indicates mean diffusivity.

* P values represent the level of significance from the Tukey test for multiple comparisons. Data are reported as mean \pm SEM

cervical spinal cord with a caudal injury are likely to be the result of changes in the cellular microstructure of the spinal cord. Preferential loss of large-diameter axons has been documented in the chronic stages of SCI, ${ }^{26}$ resulting in predominantly small axons in damaged white matter tracts and a corresponding lower $\lambda_{1}{ }^{38}$ Progressive demyelination has also been documented during the chronic stages of injury, ${ }^{8-10}$ and subsequent remyelination results in axons with significantly decreased myelin sheath thickness. ${ }^{8,25-27}$ Demyelination, combined with atrophy of the spinal cord ${ }^{11}$ in the chronic stages of SCI in regions remote from the lesion, could cause a decrease in $\lambda_{\mathrm{t}}{ }^{37,39}$ These changes in microstructure could account for the changes in diffusivity in the rostral spinal cord, but an animal study examining the diffusion parameters and corresponding histology would be needed to confirm this hypothesis.

\section{Study Limitations}

One potential limitation to this study was the use of a singleshot twice-refocused spin-echo EPI pulse sequence for obtaining DTI images of the spinal cord. Although many pulse sequences have been developed for spinal cord diffusion imaging, ${ }^{40-44}$ a recent study has suggested that these pulse sequences produce similar estimates of diffusion coefficients. ${ }^{12}$ Because of its ease of implementation in the clinical environment, many studies have demonstrated the ability of singleshot diffusion tensor EPI to estimate the diffusion characteristics of the spinal cord. ${ }^{1,5,12,45,46}$ Furthermore, the use of a twice-refocused spin-echo preparation, which is typically available on clinical MR imaging scanners, has been shown to improve significantly image quality and reduce susceptibility distortions. ${ }^{28}$

Although this study would have benefited from a larger injury-population size, the diffusion measurements observed in the lesion epicenter are similar to those previously reported in acute injury, ${ }^{7}$ and trends observed remote from the lesion are similar to those reported in recent human studies. ${ }^{13}$ Despite consistencies with similar studies, the relatively small and heterogeneous population of injured subjects with SCI is an additional limitation to the current study.

A final limitation to this study was the age range repre- sented by the normative dataset. MD has been shown to increase slightly and FA has been shown to decrease slightly with increasing age, though the magnitude of the changes was minimal and likely did not affect the results. For example, for a period of 60 years (25-85 years of age), the average MD has been shown to increase approximately $0.1 \times 10^{-3} \mathrm{~mm}^{2} / \mathrm{s}$ and the mean FA has been shown to decrease approximately $0.04{ }^{4}$ We observed changes in MD and FA in chronic SCI well beyond the magnitude expected from normal aging, and we observed no trends in the data suggesting that subject age was responsible for the observed differences between groups. To verify the absence of an age bias, we repeated the analysis eliminating both the youngest control subjects and the oldest subjects with SCI from the analysis, which resulted in similar trends in the data and similar differences between control and SCI groups. Although the magnitude of changes in diffusion characteristics is expected to change only slightly during normal aging, discretion should be used when interpreting our results because the effects of aging on this particular pathologic population have not been explored.

\section{Conclusion}

In the current study, we have demonstrated changes in DTI measurements in human chronic SCI by using a widely available clinical pulse sequence. In addition, the techniques used in this study provide clinically relevant information on lesion location and size along with the overall integrity of the spinal cord during chronic SCI.

\section{References}

1. Sagiuchi T, Iida H, Tachibana S, et al. Diffusion-weighted MRI in anterior spinal artery stroke of the cervical spinal cord. J Comput Assist Tomogr 2003;27:410-14

2. Clark CA, Werring DJ, Miller DH. Diffusion imaging of the spinal cord in vivo: estimation of the principal diffusivities and application to multiple sclerosis. Magn Reson Med 2000;43:133-38

3. Ciccarelli O, Werring DJ, Barker GJ, et al. A study of the mechanisms of normal-appearing white matter damage in multiple sclerosis using diffusion tensor imaging: evidence of Wallerian degeneration. J Neurol 2003;250:287-92

4. Mamata H, Jolesz FA, Maier SE. Apparent diffusion coefficient and fractional anisotropy in spinal cord: age and cervical spondylosis-related changes. J Magn Reson Imaging 2005;22:38-43

5. Facon D, Ozanne A, Fillard P, et al. MR diffusion tensor imaging and fiber tracking in spinal cord compression. AJNR Am J Neuroradiol 2005;26:1587-94

6. Sagiuchi T, Tachibana S, Endo M, et al. Diffusion-weighted MRI of the cervical cord in acute spinal cord injury with type II odontoid fracture. J Comput Assist Tomogr 2002;26:654-56

7. Shanmuganathan K, Gullapalli RP, Zhuo J, et al. Diffusion tensor MR imaging in cervical spine trauma. AJNR Am J Neuroradiol 2008;29:655-59

8. Totoiu MO, Keirstead HS. Spinal cord injury is accompanied by chronic progressive demyelination. J Comp Neurol 2005;486:373-83

9. Waxman SG. Demyelination in spinal cord injury. J Neurol Sci 1989;91:1-14

10. Bunge RP, Pucket WR, Becerra JL, et al. Observation on the pathology of human spinal cord injury: a review and classification of 22 new cases with details from a case of chronic cord compression with extensive focal demyelination. Adv Neurol 1993;59:75-89

11. Potter K, Saifuddin A. MRI of chronic spinal cord injury. Br J Radiol 2003;76:347-52

12. Ellingson BM, Ulmer JL, Kurpad SN, et al. Diffusion tensor MR imaging of the neurologically intact human spinal cord. AJNR Am J Neuroradiol 2008;30:1279-84

13. Ellingson BM, Ulmer JL, Schmit BD. Morphology and morphometry of human chronic spinal cord injury using diffusion tensor imaging and fuzzy logic. Ann Biomed Eng 2008;36:224-36

14. Ford JC, Hackney DB, Alsop DC, et al. MRI characterization of diffusion coefficients in a rat spinal cord injury model. Magn Reson Med 1994;31:488-94

15. Deo AA, Grill RJ, Hasan $\mathrm{K}$, et al. In vivo serial diffusion tensor imaging of experimental spinal cord injury. J Neurosci Res 2006;83:801-10

16. Nevo U, Hauben E, Yoles E, et al. Diffusion anisotropy MRI for quantitative assessment of recovery in the rat spinal cord. Magn Reson Med 2001;45:1-9 
17. Fraidakis $\mathrm{M}$, Klason $\mathrm{T}$, Cheng $\mathrm{H}$, et al. High-resolution MRI of intact and transected rat spinal cord. Exp Neurol 1998;153:299-312

18. Schwartz ED, Shumsky JS, Wehrli S, et al. Ex vivo MR determined apparent diffusion coefficients correlate with motor recovery mediated by intraspinal transplants of fibroblasts genetically modified to express BDNF. Exp Neurol 2003;182:49-63

19. Demir A, Ries M, Moonen CT, et al. Diffusion-weighted MR imaging with apparent diffusion coefficient and apparent diffusion tensor maps in cervical spondylotic myelopathy. Radiology 2003;229:37-43

20. Ito $\mathrm{T}$, Oyanagi $\mathrm{K}$, Wakabayashi $\mathrm{K}$, et al. Traumatic spinal cord injury: a neuropathological study on the longitudinal spreading of the lesions. Acta Neuropathol 1997;93:13-18

21. Nightingale EJ, Raymond J, Middleton JW, et al. Benefits of FES gait in a spinal cord injured population. Spinal Cord 2007;45:646-57

22. Scivoletto G, Ivanenko $Y$, Morganti B, et al. Plasticity of spinal centers in spinal cord injury patients: new concepts for gait evaluation and training. Neurorehabil Neural Repair 2007;21:358-65

23. Marshall L, Knowlton S, Garfin S. Deterioration following spinal cord injury: a multicenter study. J Neurosurg 1987;66:400-04

24. Piepmeier JM, Jenkins N. Late changes in neurological examinations following traumatic spinal cord injury. J Neurosurg 1988;69:399-402

25. Blakemore WF. Pattern of remyelination in the CNS. Nature 1974;249:577-78

26. Blight AR, Decrescito V. Morphometric analysis of experimental spinal cord injury in the cat: the relation of injury intensity to survival of myelinated axons. Neuroscience 1986;19:321-41

27. Harrison BM, McDonald WI. Remyelination after transient experimental compression of the spinal cord. Ann Neurol 1977;1:542-51

28. Reese TG, Heid O, Weisskoff RM, et al. Reduction of eddy-current-induced distortion in diffusion MRI using a twice-refocused spin echo. Magn Reson Med 2003;49:177-82

29. Poonawalla AH, Zhou XJ. Analytical error propagation in diffusion anisotropy calculations. J Magn Reson Imaging 2004;19:489-98

30. Jones DK, Horsfield MA, Simmons A. Optimal strategies for measuring diffusion in anisotropic systems by magnetic resonance imaging. Magnetic Reson Med 1999;42:515-25

31. Koay CG, Carew JD, Alexander AL, et al. Investigation of anomalous estimates of tensor-derived quantities in diffusion tensor imaging. Magn Reson Med 2006;55:930-36

32. Pierpaoli C, Basser PJ. Toward a quantitative assessment of diffusion anisotropy. Magn Reson Med 1996;36:893-906
33. Ellingson BM, Ulmer JL, Schmit BD. Gray and white matter delineation in the human spinal cord using diffusion tensor imaging and fuzzy logic. Acad Radiol 2007;14:847-58

34. Kaufman L, Kramer DM, Crooks LE, et al. Measuring signal-to-noise ratios in MR imaging. Radiology 1989;173:265-67

35. Schwartz ED, Chin CL, Shumsky JS, et al. Apparent diffusion coefficients in spinal cord transplants and surrounding white matter correlate with degree of axonal dieback after injury in rats. AJNR Am J Neuroradiol 2005;26:7-18

36. Takahashi M, Zhang G, Selzer ME, et al. Diffusion MR Studies Characterize Axonal Degeneration and Dieback in the Injured Larval Sea Lamprey Spinal Cord: Proceedings of the International Society of Magnetic Resonance in Medicine, Glasgow, Scotland, April 21-27, 2001; Glasgow, Scotland: ISMRM; 2001:1518

37. DeBoy CA, Zhang J, Dike S, et al. High-resolution diffusion tensor imaging of axonal damage in focal inflammatory and demyelinating lesions in rat spinal cord. Brain 2007;130:2199-210

38. Schwartz ED, Cooper ET, Fan Y, et al. MRI diffusion coefficients in spinal cord correlate with axon morphometry. Neuroreport 2005;16:73-76

39. Ford JC, Hackney DB. Numerical model for calculations of apparent diffusion coefficient (ADC) in permeable cylinders: comparison with measured ADC in spinal cord white matter. Magn Reson Med 1997;37:387-94

40. Bammer R, Herneth AM, Maier SE, et al. Line Scan Diffusion Imaging of the Spine: Proceedings of the International Society of Magnetic Resonance in Medicine, Honolulu, Hawaii, May 18-24, 2002. Honolulu, Hawaii: ISMRM; 2002:10

41. Holder CA, Muthupillai R, Mukundan S Jr, et al. Diffusion-weighted MR imaging of the normal human spinal cord in vivo. AJNR Am J Neuroradiol 2000;21:1799-806

42. Cercignani M, Horsfield MA, Agosta F, et al. Sensitivity-encoded diffusion tensor MR imaging of the cervical cord. AJNR Am J Neuroradiol 2003;24:1254-56

43. Bammer R, Fazekas F, Augustin M, et al. Diffusion-weighted MR imaging of the spinal cord. AJNR Am J Neuroradiol 2000;21:587-91

44. Bammer R, Stollberger R, Augustin M, et al. Diffusion-weighted imaging with navigated interleaved echo-planar imaging and a conventional gradient system. Radiology 1999;211:799-806

45. Nagayoshi K, Kimura S, Ochi M, et al. Diffusion-weighted echo planar imaging of the normal human cervical spinal cord. J Comput Assist Tomogr 2000;24:482-85

46. Kuker W, Weller M, Klose U, et al. Diffusion-weighted MRI of spinal cord infarction: high-resolution imaging and time course of diffusion abnormality. J Neurol 2004;251:818-24 